\title{
Automated Driving Test System
}

\author{
Rashmi Konapanavar*, Madhuri Deshapande **, Kishor Hawaladar***, \\ MahammadMalikRihan Rajagoli****, Prof. Anand Konnur \\ ( Department of Electronics \& Communication, KLE Dr. M.S.Sheshgiri College of Engineering \& Technology, \\ Belagavi, Visvesvaraya Technological University, India.)
}

\begin{abstract}
This paper presents about the automation of driving license test system. Normally, in driving test a candidate applied for license have to drive over a closed loop path in front of the authorities. The candidate has to drive over the path with specific rules and if he fails to do he will be disqualified. For that, the authorities watch candidate manually. In this project, the arduino system with sensors has been developed for watching the candidate for getting license. By using this, the candidate who fails to attain criteria in the vehicle on track, the sensors can be monitored then it was processed by another Arduino system connected to laptop or PC. The registered person entering for license test will be authenticated by using finger print sensor. So that they will automatically select or reject by the system.
\end{abstract}

Keywords: Arduino, Driving track, Fingerprint sensors,HC-12, IR sensors.

\section{INTRODUCTION}

In recent days life technology has been developed and the growing technology introduces many advances in day to day life. This project helps to get driving license for the candidate without any biased interference of authorities. The automated driving license test track is designed in all the possible driving challenges on the track that one might face on the road. It assesses the overall traffic knowledge and driving skills of applicants on the automated track through sensors. In this project IR sensors are used on the track to monitor the vehicle movement and tests driving skills of the candidate. And also the authority person needs not to monitor the candidate who enters for the license test. Before entering for the driving license test candidate has to be registered and authenticated using fingerprint sensor. During driving the person is monitored by the sensors. The Arduino monitors various sensors and verifies sensor readings with preset criteria and generates the results, which will be displayed on the computer. If candidate gets qualified he can get license. Suppose if the candidate fails to drive properly then that test is postpone for next particular day.

\section{LITERATURE SURVEY}

In day to day life many modern transporting vehicles coming in to the market. As like population Increases the usage of these vehicles also increases in a wider scale. Most of them prefer for four wheeler transporting systems. For operating the vehicles the license is compulsory. Normally, in driving test a candidate applied for license have to drive over a closed loop path in front of the authorities. The corruption in the current system is very much; to tackle the corruption we came up with this idea. As the level of transparency increases, the prevalence rate of corruption decreases in issuing of driving licences in the RTOs. It does not require interaction between the officials and the citizens, negating the chances of corruption.

The comparative analysis of the numbers of driving licences issued by the Karnataka Transport Department indicates a sharp decline. Compared to 36,442 driving licences issued during Sep 2009 to March 2010, the Department issued only 25,796driving licences during March 2010 to September 2010. It indicates that the process of issuing driving licences has become stringent leaving less chances of indulging in both demand and supply side of corruption.

\section{COMPONENT DESCRIPTION 3.1 Arduino Uno R3}

This is the Arduino Uno R3. The Uno uses an ATmega328P found on the Uno. This allows for faster transfer rates and more memory. No drivers needed for Linux or Mac (.inf file for Windows is needed and included in the Arduino IDE), and the ability to have the Uno show up as a keyboard, mouse, joystick, etc. In addition, there are two new pins placed near the RESET pin. One is the IOREF that allow the shields to adapt to the voltage provided from the board. The other is a not connected and is reserved for future purposes. The Uno R3 works with all existing shields but can adapt to new shields which use these additional pins.

The Arduino Uno is a microcontroller board based on the ATmega328. Arduino is an open- 
source, prototyping platform and its simplicity makes it ideal for hobbyists to use as well as professionals. The Arduino Uno has 14 digital input/output pins (6 can be used as PWM outputs), 6 analog inputs, a $16 \mathrm{MHz}$ crystal oscillator, a USB connection, a power jack, an ICSP header, and a reset button. It contains everything needed to support the microcontroller; simply connect it to a computer with a USB cable or power it with a AC-to-DC adapter or battery to get started. The Arduino Uno differs from all preceding boards in that it does not use the FTDI USB-to-serial driver chip. Instead, it features the Atmega328P microcontroller chip programmed as a USB-to-serial converter.

\subsection{HC-12 Transceiver}

The HC-12 is a half-duplex wireless serial communication module with 100 channels in the 433.4-473.0 $\mathrm{MHz}$ range that is capable of transmitting up to $1 \mathrm{~km}$. This project will begin by using the HC-12 to create a wireless link between two computers and end with a second article that creates a simple wireless GPS tracker.

The HC-12 is a half-duplex $20 \mathrm{dBm}(100$ $\mathrm{mW}$ ) transmitter paired with a receiver that has -117 $\mathrm{dBm}\left(2 \times 10^{-15} \mathrm{~W}\right)$ sensitivity at 5000 bps. Paired with an external antenna, these transceivers are capable of communicating up to and possibly slightly beyond 1 $\mathrm{km}$ in the open and are more than adequate for providing coverage throughout a typical house. Fig 1 shows a HC-12 transceiver module.

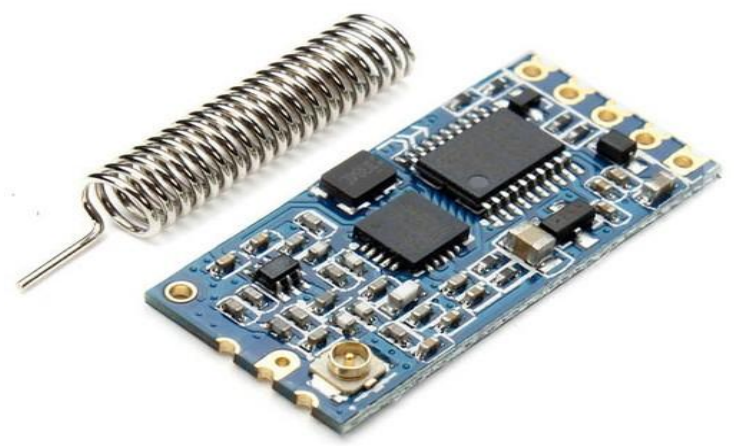

fig 1. hc-12 transceiver module

Combined with other components, the Si4463 and STM8S003 create the HC-12 transceiver, which provides a 4-pin TTL-level UART interface (Vcc, Gnd, Tx, Rx), with a 5th pin that is used to enter "command" mode for changing the module's configuration. The HC-12 has 100 supported channels spaced $400 \mathrm{kHz}$ apart, eight transmit levels, eight supported baud rates, and three different working modes. The 5th pin on the HC-12 is labelled "Set" and, when driven to logic low, allows various settings to be selected on the HC-12 using AT commands sent to the "RXD" pin. The default configuration of the $\mathrm{HC}-12$ is FU3-on
Channel 1, FU3 is a fully automatic and transparent (to other devices) setting that adapts to the transmission rate of the connected device (although 9600 baud is still required to program it in Command mode).

\subsection{IR Proximity Sensor}

A proximity sensor is a sensor able to detect the presence of nearby objects without any physical contact. A proximity sensor often emits an electromagnetic field or a beam of electromagnetic radiation (infrared, for instance), and looks for changes in the field or return signal. The object being sensed is often referred to as the proximity sensor's target. Fig. 2 shows IR proximity sensor. Different proximity sensor targets demand different sensors. For example, a capacitive or photoelectric sensor might be suitable for a plastic target; an inductive proximity sensor always requires a metal target. The maximum distance that this sensor can detect is defined "nominal range". Some sensors have adjustments of the nominal range or means to report a graduated detection distance. Some know this process as "thermo-sensation". Proximity sensors can have a high reliability and long functional life because of the absence of mechanical parts and lack of physical contact between sensor and the sensed object.

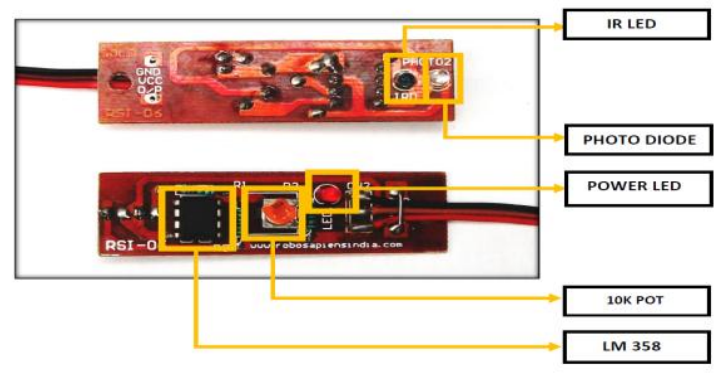

fig. 2 ir proximity sensor

Proximity sensors are commonly used on smart-phones to detect (and skip) accidental touchscreen taps when held to the ear during a call. They are also used in machine vibration monitoring to measure the variation in distance between a shaft and its support bearing. This is common in large steam turbines, compressors, and motors that use sleeve-type bearings. International Electro-technical Commission (IEC) 60947-5-2 defines the technical details of proximity sensors.

\subsection{Fingerprint Sensor}

This is an optical biometric fingerprint reader/sensor (R305) module with TTL UART interface for direct connections to a microcontroller UART. The user can store the finger print data in the module and can configure it in 1:1 or $1: \mathrm{N}$ mode for identifying the person. This module can directly 
interface with any $3.3 \mathrm{~V}$ or $5 \mathrm{~V}$ microcontrollers, but a suitable level converter/serial adapter is required for interfacing with the serial port of a PC. By way of the serial interface, fingerprint reader/sensor module can communicate with a microcontroller $(\mathrm{uC})$ runs on of $3.3 \mathrm{~V}$ or $5 \mathrm{~V}$ power supply. TX/TD pin of the module connects with RXD (RX-IN pin of the $\mathrm{uC}$ ), and RX/RD pin connects with TXD (TXOUT pin of the microcontroller).

Fingerprint processing includes two parts, fingerprint enrolment and fingerprint matching (the matching can be $1: 1$ or 1:N). When enrolling, user needs to enter the finger two times. The system will process the two time finger images, generate a template of the finger based on processing results and store the template. When matching, user enters the finger through optical sensor and system will generate a template of the finger and compare it with templates of the finger library. For 1:1 matching, system will compare the live finger with specific template designated in the Module; for $1: \mathrm{N}$ matching, or searching, system will search the whole finger library for the matching finger. In both circumstances, system will return the matching result, success or failure.

\section{SYSTEM DESIGN}

Fig. 3 shows the block diagram of the system. The system consists of two parts 1 . Control Unit and 2. Vehicle Monitoring. Control unit consist of Fingerprint sensor for authentication of the candidate and IR sensors to monitor the start and end of the test. The IR sensors here are placed on the track. Vehicle monitoring unit is fitted on the vehicle, it consist of the IR proximity sensors, which will check that vehicle is moving in the specified track.

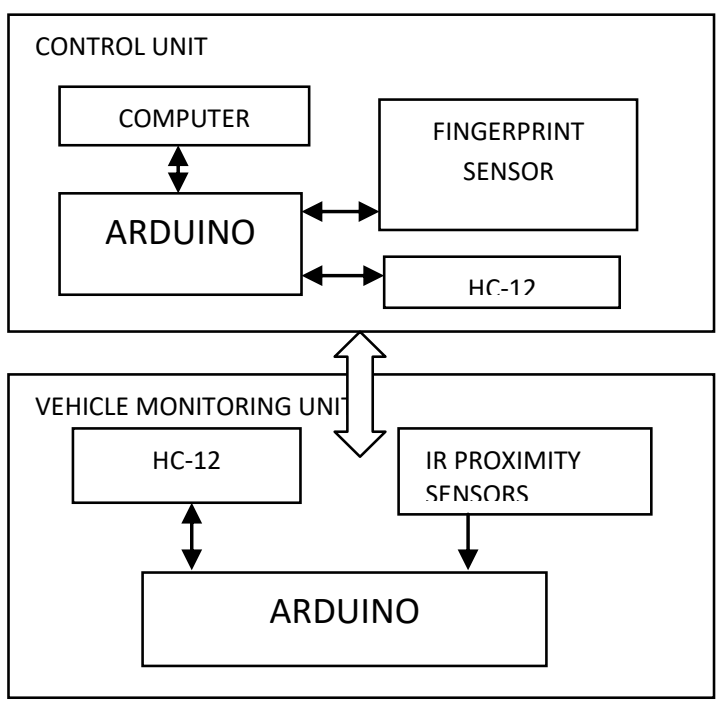

fig3. block diagram
The Arduino is used here to control the demo vehicle. Both the units communicate with each other using $\mathrm{HC} 12$ transmitter and receiver pair. The system is designed to monitor the driving skills of the candidate using Arduino. The system works as follows,

- The candidate has to register before taking the test, the candidate is authenticated using fingerprint.

- At the time of the test the candidate is authenticated using fingerprint. After the authentication is successful the candidate can proceed for test.

- An IR sensor is used to check the vehicle is started to move or not, as the vehicle moves from its initial position the test begins.

- The proximity IR sensors are placed on the both sides of the vehicle to check that vehicle is moving in specified path.

- When the vehicle moves out of the specified track a pulse is generated and is recorded by Arduino. When vehicle completes the track and comes to end line, the test ends.

- After the test ends the Arduino on the vehicle sends data to another Arduino which is connected to the computer using the Zigbee transmitter and receiver pair.

- This data is compared with predefined parameters. And based on the comparison the candidate is issued license or asked to retake the test in near future.

\section{RESULTS \& DISCUSSION}

The system we designed and tested can be used for the following applications with little modifications. The advantages and disadvantages are discussed below. The snapshots of the project prototype and results are added below The snapshot of the serial monitor window of Arduino IDE is shown below which shows the results of the driving conducted for a registered candidate

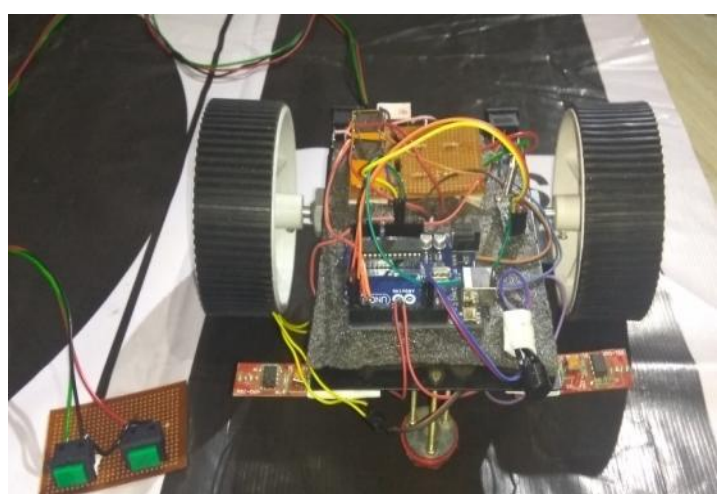

Fig 4 vehicle prototype 

ISSN : 2248-9622, Vol. 7, Issue 7, ( Part -2) July 2017, pp.46-49

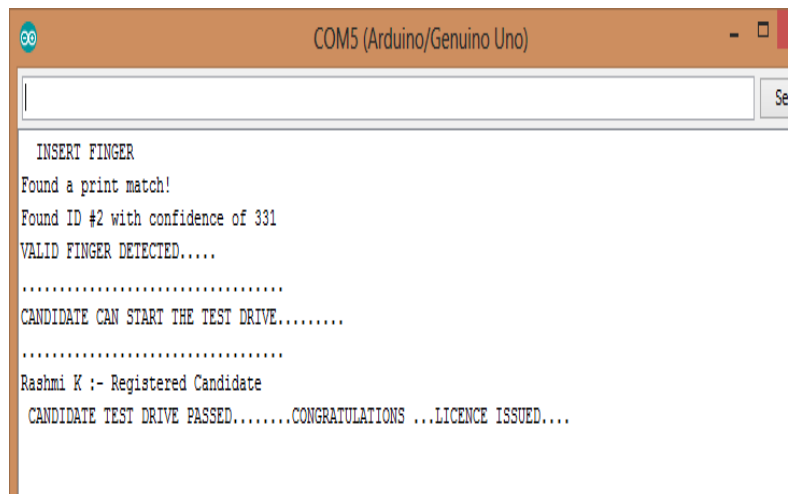

fig. 5 results

\subsection{Applications:}

- In RTO Driving test zones.

- Private practice zones

\subsection{Advantages:}

- Ensuring transparency in the system by tackling corruption.

- Promoting quality drivers.

- Unbiased results.

5.3 Disadvantages:

- Complex system design.

- Dark sensor may give incorrect readings.

\section{CONCLUSION}

Monitoring the candidate's driving skills who applied for driving license whether the candidate is eligible for getting license by using Arduino and also the system is used to test driving skills on vehicle without any authorities. So that candidate will be automatically selected or reject by the system. It will reduce the manpower and there is no chance for any illegal activity.

\section{ACKNOWLEDGEMENTS}

We would like to express my sincere thanks to our Head of Dept. Prof. S.B. Kulkarni for endowing us with his valuable time and knowledge. I would like to thank our project guide Prof. Anand Konnur for his keen interest and concern towards our project.

\section{REFERENCES}

[1] Martin Albert, Alexander Lange, Annika Schmidt, Martin Wimmer, Klaus Bengler, "Automated driving - Assessment of interaction concepts under real driving conditions", 6th International Conference on Applied Human Factors and Ergonomics (AHFE 2015) and the Affiliated Conferences, AHFE 2015.

[2] D.Sarathkumar, C.K Sathish Kumar, S.Nithya, E.Thilagavathi, "Automatic Two Wheeler Driving Licence System by Using Labview", International Journal of Advanced
Research in Electrical, Electronics and Instrumentation Engineering, Vol. 5, Issue 4, April 2016.

[3] Prince Samuel S, Kiruba R, Saranya M, "Development of Test RIG for Automated Driving Test Track and Issuing License Using LabVIEW", International Journal on Recent and Innovation Trends in Computing and Communication, Volume: 3 Issue: 12.

[4] Ms.Suvarna A.Dodke, "AUTOMATION OF DRIVING LICENSE TEST USING WIRELESS SENSOR NETWORK", International Research Journal of Engineering and Technology (IRJET) Volume: 02 Issue: 08 Nov-2015 